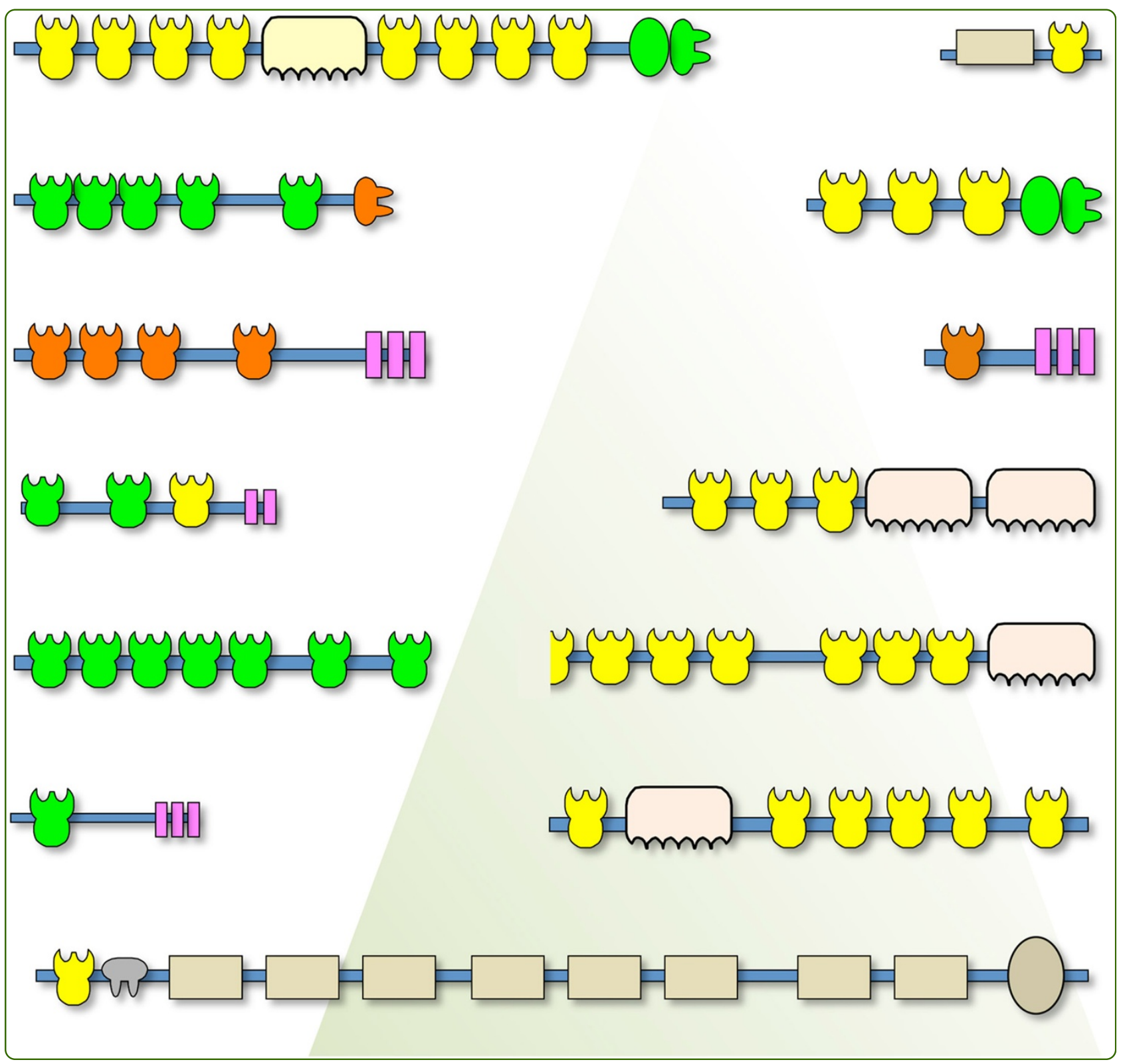

\title{
Cellulosomics of the cellulolytic thermophile Clostridium clariflavum
}

Artzi et al. 


\title{
Cellulosomics of the cellulolytic thermophile Clostridium clariflavum
}

\author{
Lior Artzi ${ }^{1}$, Bareket Dassa', llya Borovok², Melina Shamshoum, Raphael Lamed ${ }^{2}$ and Edward A Bayer ${ }^{1 *}$
}

\begin{abstract}
Background: Clostridium clariflavum is an anaerobic, thermophilic, Gram-positive bacterium, capable of growth on crystalline cellulose as a single carbon source. The genome of $C$. clariflavum has been sequenced to completion, and numerous cellulosomal genes were identified, including putative scaffoldin and enzyme subunits.

Results: Bioinformatic analysis of the C. clariflavum genome revealed 49 cohesin modules distributed on 13 different scaffoldins and 79 dockerin-containing proteins, suggesting an abundance of putative cellulosome assemblies. The 13-scaffoldin system of C. clariflavum is highly reminiscent of the proposed cellulosome system of Acetivibrio cellulolyticus. Analysis of the C. clariflavum type I dockerin sequences indicated a very high level of conservation, wherein the putative recognition residues are remarkably similar to those of $A$. cellulolyticus. The numerous interactions among the cellulosomal components were elucidated using a standardized affinity ELISA-based fusion-protein system. The results revealed a rather simplistic recognition pattern of cohesin-dockerin interaction, whereby the type I and type II cohesins generally recognized the dockerins of the same type. The anticipated exception to this rule was the type I dockerin of the ScaB adaptor scaffoldin which bound selectively to the type I cohesins of ScaC and ScaJ.
\end{abstract}

Conclusions: The findings reveal an intricate picture of predicted cellulosome assemblies in C. clariflavum. The network of cohesin-dockerin pairs provides a thermophilic alternative to those of $C$. thermocellum and a basis for subsequent utilization of the C. clariflavum cellulosomal system for biotechnological application.

Keywords: Cellulosomes, Cellulases, Cohesin, Dockerin, Scaffoldin, CBM, Glycoside hydrolases, Biomass degradation, Biofuels

\section{Background}

In today's world, the plant cell wall is one of industry's most common raw materials and provides the main component of fabric, paper, and wood. These materials, as well as byproducts from agriculture eventually end up as cellulosic waste and are a major source of pollution [1,2]. The plant cell wall contains a variety of polysaccharides, including cellulose as the major component. Cellulose is a crystalline polysaccharide, which is constructed from glucose monomers linked together by $\beta$-1,4-linkages [3-6]. An efficient way to degrade cellulose to single glucose molecules will lead to potential recycling of the cellulose and conversion of the glucose subunits to bioethanol and/or other useful chemicals by a simple fermentation step. Today, we rely on fossil fuels as a primary energy

\footnotetext{
*Correspondence: ed.bayer@weizmann.ac.il

'Department of Biological Chemistry, The Weizmann Institute of Science, Rehovot, Israel

Full list of author information is available at the end of the article
}

source, and the ability to harvest the energy encapsulated in biomass can help liberate society from the complete dependence on unsustainable fuel sources [7-9].

The cellulosome is a high-molecular-weight, multienzyme complex, found in anaerobic bacteria and has the ability to efficiently degrade cellulosic substrates [6,10-12]. Cellulosomes are secreted from the bacterial cell and may then be anchored to the cell surface or found in the free state in the extracellular medium. The cellulosome was first discovered in the anaerobic, thermophilic bacterium Clostridium thermocellum [13-15]. It is composed of two types of protein components: the structural proteins (scaffoldins) and the enzymatic subunits. The scaffoldins are non-catalytic proteins that carry cohesin modules, which are responsible for the integration of the enzyme subunits into the complex $[10,12,16]$. Scaffoldins that bind enzymes are called primary scaffoldins, and they usually contain type I cohesins [17-21]. Cellulolytic enzymes contain a type I dockerin 
module that interacts specifically with a type I cohesin module found on the scaffoldin. In this way, the enzymes can integrate into the scaffoldins and create the large multi-enzyme cellulosome complexes. Some of these primary scaffoldins contain a dockerin module, which gives them the ability to assemble with another scaffoldin called the adaptor scaffoldin, first seen in the bacterium Acetivibrio cellulolyticus [22]. This arrangement allows attachment of the cellulosome to the cell surface via successive interactions, first between the primary and adaptor scaffoldins, and then via a cell-anchoring scaffoldin. The primary scaffoldin may also interact directly with the anchoring scaffoldin that fastens the cellulosome to the cell surface via an S-layer homology (SLH) domain [23,24]. This architectural flexibility multiplies the possible enzyme compositions of the cellulosome [12]. In A. cellulolyticus, the adaptor scaffoldin bears type II cohesins that specifically interact with the type II dockerin of the primary scaffoldin. In addition, a scaffoldin may contain a carbohydratebinding module (CBM) that allows the cellulosome to target specific carbohydrate substrates [20,25-28]. The scaffoldins and enzymes are the building blocks of the cellulosome, and the specific cohesin-dockerin interactions give rise to extensive assemblage possibilities and a variety of complexes.

Clostridium clariflavum is a gram-positive, anaerobic, thermophilic, spore-forming bacterium that was first discovered and isolated from an anaerobic sludge taken from a thermophilic methanogenic bioreactor. It has shown the ability to utilize cellulose and cellobiose, the only source of carbon and energy [29-31]. Studies of $16 \mathrm{~S}$ rRNA-based phylogenetic have revealed that C. clariflavum and C. thermocellum are closely related. Interestingly, another closely related species to C. clariflavum is $A$. cellulolyticus, an anaerobic, mesophilic, cellulolytic bacterium, with a complicated cellulosomal system containing 16 scaffoldins and 143 putative dockerin-containing proteins [32-34]. These properties of C. clariflavum render it of prime interest for further exploration, and motivate us to reveal its cellulosome system and enzymes. Discovery of novel, potent cellulolytic enzymes and cellulosomes from these bacterial species may help the development of methods for efficient degradation of cellulose. The entire C. clariflavum genome was sequenced and putative enzymes (cellulosomal and non-cellulosomal) were revealed, such as bifunctional glycoside hydrolases with or without a dockerin module, and putative scaffoldins [31].

In the current study, we investigated the genes that include presumed cellulosomal modules (for example, cohesins, dockerins and CBMs). Using DNA sequence data, we were able to bioinformatically characterize dockerin-bearing proteins and cohesin-containing scaffoldins of C. clariflavum, and compare them with the cellulosomal proteins of $C$. thermocellum and A. cellulolyticus.
Recombinant cohesin and dockerin modules that were identified from the C. clariflavum genome were cloned into matching fusion-protein cassettes and expressed. The modules served for evaluation of the various cohesindockerin interactions which then allowed us to predict potential cell-bound and cell-free cellulosomal complexes in this newly described cellulosome-producing bacterium.

\section{Results}

\section{Variety of cohesin-containing proteins}

Recently, the $4.9 \mathrm{Mbp}$ genome of C. clariflavum DSM 19732 was sequenced and annotated [31]. We further investigated the presumed cohesin-containing proteins and identified 49 cohesin modules distributed among 13 different scaffoldins (Figure 1), some of which carry both dockerin and cohesin modules on the same protein. Among these modules, two cohesins seem to be truncated. The other 47 complete putative cohesin sequences were aligned with cohesins from $A$. cellulolyticus and $C$. thermocellum (Additional file 1: Figure S1) and are presented on a phylogenetic tree (Figure 2), divided into type I and type II cohesins by sequence. Fifteen cohesin modules are classified as type II and 34 cohesin modules are classified as type I. The type I cohesin modules are separated into two groups, which may suggest the division of the type I group into subtypes. The putative C. clariflavum cohesin sequences are closely related to those of $A$. cellulolyticus on the phylogenetic tree. For the majority of the cohesin modules of $A$. cellulolyticus there is a homolog in the $C$. clariflavum genome. In contrast, the cohesin modules of $C$. thermocellum are clustered on a separate branch of the tree and are in general more distantly related to the A. cellulolyticus and C. clariflavum modules.

\section{Architecture and modular arrangement of the scaffoldins}

The modular organization of the cohesin and dockerin modules on the 13 different scaffoldins is represented in Figure 1. The names of the scaffoldins are based on the homology of the cohesin modules of C. clariflavum to the cohesin modules from $A$. cellulolyticus, according to Dassa et al. [34]. The scaffoldins possess a signal peptide at their N-terminus (except ScaO and ScaM(a)), suggesting that the scaffoldins are secreted from the cell. Most of the scaffoldins carry only one type of cohesin (type I or type II), except ScaD which contains both types of cohesins: the first two ScaD cohesins are type II, and the third is type I. This unique type of scaffoldin is very similar to the ScaD scaffoldin of $A$. cellulolyticus [ZP_09464030] [31,37]. In addition, ScaD contains two repeats of SLH domains, which enable its anchoring to the cell wall.

Likewise the homology between other scaffoldins of $C$. clariflavum and A. cellulolyticus is high, and the modular organization of the proteins is very similar. For 


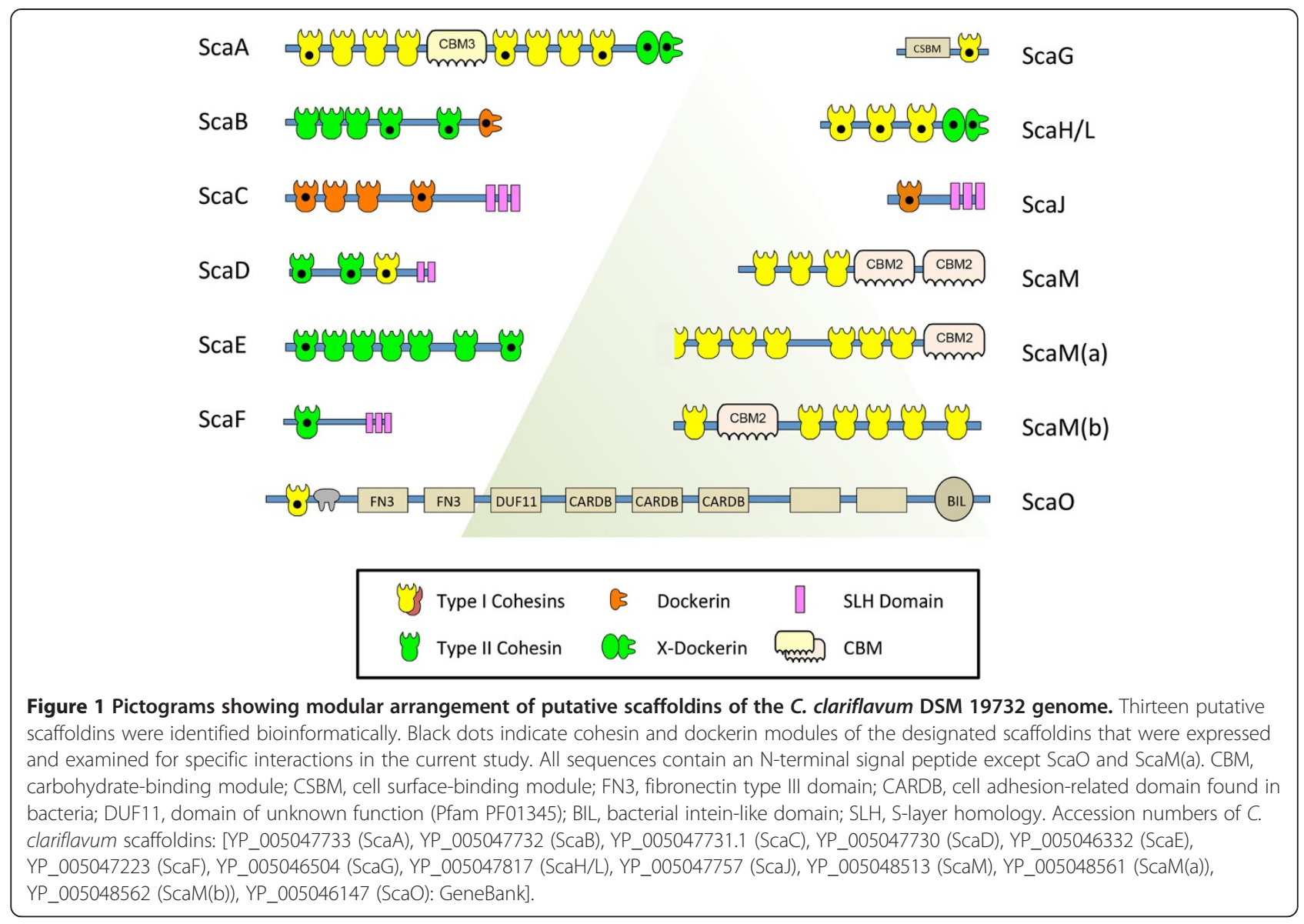

example, the primary scaffoldin ScaA is similar to ScaA [ZP_09464033.1] of A. cellulolyticus [21] and to CipA [CAA47840] of $C$. thermocellum. Eight type I cohesins are located in its sequence, like the ScaA of A. cellulolyticus (7 type I cohesins) and CipA (9 type I cohesins) of C. thermocellum, and all contain a CBM3 module (Dassa et al. 2012 [34]). At the C-terminus of the three scaffoldins there is an X-dockerin (XDoc) modular dyad, which was shown previously to bind type II cohesins [22,38-41]. In fact the C. clariflavum ScaA parallels closely ScaA of A. cellulolyticus, both in its overall modular architecture (Figure 1 and Dassa et al. [34]) and in the sequences of its various cohesin modules (Figure 2); the major difference being the unique presence of an $\mathrm{N}$-terminal catalytic module (family 9 cellulase) in the A. cellulolyticus scaffoldin, which is lacking in C. clariflavum ScaA, and instead contains an extra cohesin in the same position.

Most of the scaffoldins from C. clariflavum have a homologous scaffoldin in A. cellulolyticus. Notably, the adaptor scaffoldin $\mathrm{ScaB}$ and cell-anchoring scaffoldin ScaC have equivalent proteins in A. cellulolyticus [ZP_09464032 (ScaB) and ZP_09464031 (ScaC)]. ScaE consists exclusively of seven type II cohesins, which are closely related to the seven cohesin modules of ScaE from A. cellulolyticus [ZP_09465494] and Cthe_0736 from C. thermocellum.

ScaG has a single type I cohesin and a region annotated as a copper-amine-oxidase-like domain. Intriguingly, both A. cellulolyticus and C. thermocellum genomes include scaffoldins that are composed of the same modular type, that is, ScaG [ZP_09464788] and OlpC [YP_001036883], respectively. Interestingly, Pinheiro et al. [42] have demonstrated that the 'copper-amine-oxidase-like domain' of C. thermocellum $\mathrm{OlpC}$ is responsible for binding to the secondary cell wall polymers that are bound to the S-layer in gram-positive bacteria, thereby allowing the anchoring of OlpC to the cell wall of $C$. thermocellum. From the sequence similarity of this domain in OlpC and ScaG, it therefore seems likely that this domain in ScaG would exhibit the same cell-surface anchoring function, and the domain is thus designated cell surface-binding module (CSBM).

In addition to ScaG, there are two additional scaffoldins that are composed of a single cohesin module and a cell-anchoring module. In this context, ScaF consists of a type II cohesin and three SLH domain repeats. ScaJ, however, contains a type I cohesin and also three SLH domain repeats. ScaH/L has three type I cohesins which 


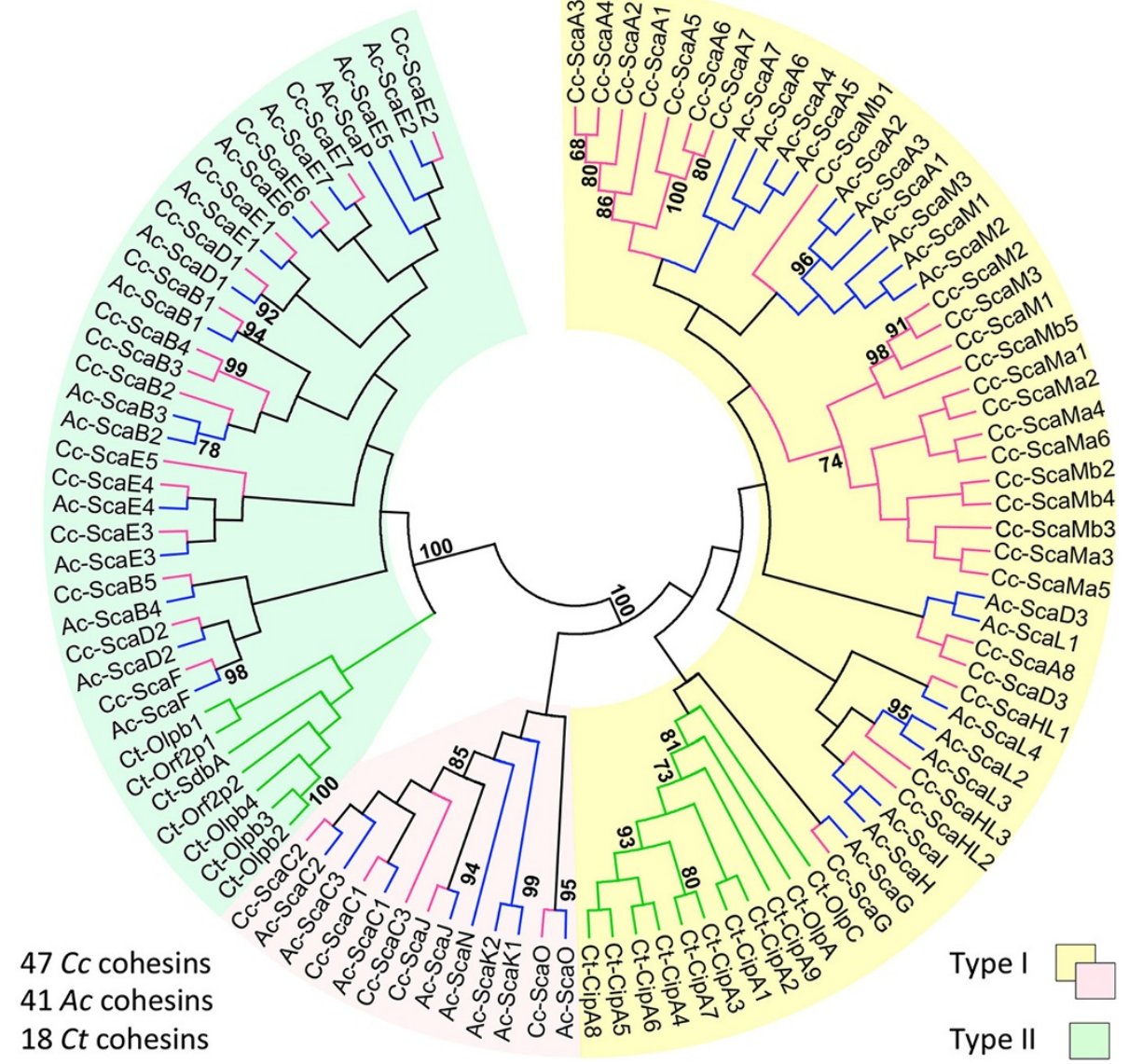

Figure 2 Phylogeny of $\boldsymbol{C}$. clariflavum cohesins. A set of 47 C. clariflavum (Cc), 41 A. cellulolyticus (Ac), and 18 C. thermocellum (Ct) cohesin-like modules, derived from deduced amino-acid sequences (supporting Additional file 1: Figure S1), was aligned using the CLUSTALW2 program at the EBI website [35], which then served to reconstruct an unrooted phylogenetic tree by the MEGA5.10 software [36], using the neighbor-joining method with 500 bootstrap replicates. Numerical values above the nodes indicate bootstrap percentiles. The cohesin-like modules distribute into two major classes: type I (yellow) and type II (green). Among the type I cohesin-like modules one subgroup is separated from the majority of the modules (pink).

are related phylogenetically to the cohesins on $\mathrm{ScaH}$ [ZP_09462752] and ScaL [ZP_09464968] of A. cellulolyticus (Dassa et al. [34], Figure 2).

C. clariflavum possesses three scaffoldins with CBM2 modules and type I cohesins (ScaM, ScaM(a), ScaM(b)). Previously, only ScaM from A. cellulolyticus [ZP_09463433] was reported to be a unique scaffoldin that bears CBM2 modules [34]. All other previously described scaffoldins contain CBM modules from family 3. Family-2 CBMs are usually attached to enzymes and bind to various polysaccharides such as cellulose and xylan [43]. In this case, ScaM of C. clariflavum is similar to ScaM of A. cellulolyticus [ZP_09463433] with its three type I cohesins and two CBM2 modules. Moreover, the cohesins of the three scaffoldins ScaM, ScaM(a) and ScaM(b) are phylogenetically related to the A. cellulolyticus ScaM cohesins (Figure 2).

The pair of ORFs, ScaM(a) and ScaM(b) was found in a unique arrangement on the $C$. clariflavum genome.
The ORF of Clocl_4212 (ScaM(b), [YP_005048562] codes for a protein with a signal peptide, at least six cohesin modules and a CBM2 module. The ORF seems to be truncated, because it ends with an $\mathrm{N}$-terminal half of a cohesin, while the second ORF, Clocl_4211 (ScaM(a), [YP_005048561] starts with a complementary C-terminal half of the cohesin (and no signal peptide), having at least six cohesins and a C-terminal CBM2 module. Both ORFs overlap on the genome, suggesting that they may reflect a single extended ORF, which underwent a frame shift. In addition, the nature of these ORFs is remarkably repetitive due to the close similarity (near-identity) of the cohesin modules, which did not allow us to validate the transcript of this locus by PCR.

Finally, a unique scaffoldin, $\mathrm{ScaO}$, bears a type I cohesin and a type I dockerin at the $\mathrm{N}$-terminus of the protein. This protein does not contain a signal peptide, which suggests it is not secreted from the bacterium, but does not 
rule out the possibility for secretion. ScaO has two putative fibronectin type III domains, three cell adhesionrelated domains, and a bacterial intein-like domain. The rest of the protein includes unknown domains. The designation of this protein is in accordance to the architectural similarity of its $\mathrm{N}$-terminal portion with portions of ScaO from A. cellulolyticus.

\section{Dockerin-containing proteins}

A large set of genes encoding 79 dockerin-containing proteins is present in the C. clariflavum genome; 75 of them have type I dockerins whereas four possess type II dockerins, which, similar to $C$. thermocellum and A. cellulolyticus, have an X-module upstream of the dockerin module. The 75 type I dockerin-containing proteins have a variety of predicted catalytic units that are distributed on 48 dockerin-containing enzymes. These enzymes include 41 glycoside hydrolases (GHs) from 15 different families, 14 carbohydrate esterases (CEs) and 2 polysaccharide lyases (PLs), whereby some of the dockerin-containing enzymes contain more than one catalytic module and are thus bifunctional [31]. Some of the dockerin-containing proteins can be classified as non-catalytic components, for example, the serpin- (Clocl_3968) and expansin-containing proteins (Clocl_1298 and Clocl_1862). In others, CBMs are the only identifiable modular type, and many others contain modules of unknown function; hence these latter dockerincontaining proteins cannot currently be classified as enzymes. Multiple sequence alignment of the dockerins and the annotated modules located in each parent protein can be found in Additional file 2: Figure S2.

Sequence conservation of the dockerin was demonstrated by performing multiple sequence alignment of the 75 type I dockerins, and creating a sequence logo of the two repeats of the dockerin modules (Figure 3). Most of the dockerin-containing proteins have two repeats of the duplicated sequence, whereby each repeat contains a predicted $\mathrm{Ca}^{+2}$-binding loop and an alpha-helix, with a linker separating the two repeats. Only one protein, Clocl_2271, has a single repeat of dockerin module, located at the Nterminus of the protein. Notably, the $\mathrm{Ca}^{+2}$-binding repeats are highly conserved in both repeats and the coordinating residues are located at positions 1, 3, 5, 9 and 12 . The predicted residues critical for cohesin-dockerin recognition are residues 10,11,17, 18, and 22 [44-46]. Most of the latter residues are highly conserved in $C$. clariflavum, except residue 18 which is variable. Interestingly, the predicted recognition residues of the $C$. clariflavum dockerins are highly similar to those of $A$. cellulolyticus. Three of the dominant residues are identical (S, I and G in positions 10, 11 and 22) and a fourth very similar (K versus $\mathrm{R}$ in position 17 of $C$. clariflavum and A. cellulolyticus, respectively).

Significantly, the predicted recognition residues of the type I ScaB dockerin is different from all other type I dockerins in C. clariflavum. Notably, its sequence is remarkably similar to that of the ScaB dockerin of $A$. cellulolyticus (Figure 4). Moreover, the predicted recognition residues (I, N, R, D, G of the designated positions) are identical between the two sequence repeats and between the two species.

\section{Selection and design of cohesin- and dockerin-modules for interaction studies}

The multiplicity and complexity of the cellulosomal components of $C$. clariflavum enables diverse architectural assemblies of the cellulosome. In order to identify and characterize the relevant interactions among the cellulosomal components, we employed the matching fusion-protein system and affinity-based ELISA approach, developed previously in our laboratory [47]. For this purpose, we chose representative cohesin and dockerin modules and expressed them in two different cassettes: each cohesin module was $\mathrm{N}$-terminally fused to a CBM3a module originating from the CipA scaffoldin of C. thermocellum [26]. Within the context of the present work, this type of chimera is termed CBM-Coh. The counterpart - the dockerin module - was fused to the C terminus of xylanase T6 from Geobacillus stearothermophilus [48], and this type of chimera is herein termed XynDoc for the type I dockerins and Xyn-XDoc for the type II dockerins. This fusion protein system was originally developed with the purpose of achieving high-level protein expression, and for increasing the stability and solubility of the cohesin and dockerin modules. Both the thermostable xylanase T6 and CBM3a have indeed been shown to elevate expression levels in Escherichia coli cells and assist in protein solubility. The CBM3a module also allows efficient purification via its cellulose affinity properties [26]. Following protein expression, SDS-PAGE analysis of the purified proteins revealed single protein bands in agreement with their calculated molecular mass (data not shown).

The cohesins that were selected for expression are shown in Figure 1 and are labeled with a black dot. Nineteen cohesins were expressed in order to detect interactions with various dockerins. The cohesins that were expressed are as follows (enumerated from the $\mathrm{N}$ to the $\mathrm{C}$-terminus of the given protein): cohesins 1,5 and 8 from ScaA; cohesins 4 and 5 from ScaB; cohesins 1 and 4 from ScaC; cohesins 1 and 7 of ScaE; the single cohesins of ScaF, ScaG, ScaJ and ScaO; and all three cohesins of ScaD and ScaH/L (Figure 1).

Four dockerin modules were selected for expression. Three of these dockerins were from the scaffoldins: two type II dockerins were taken from ScaA and ScaH/L, along with their N-terminal X modules. A type I dockerin was taken from $\mathrm{ScaB}$, and another dockerin was taken from the GH48 enzyme [YP_005048367.1] of C. clariflavum. 


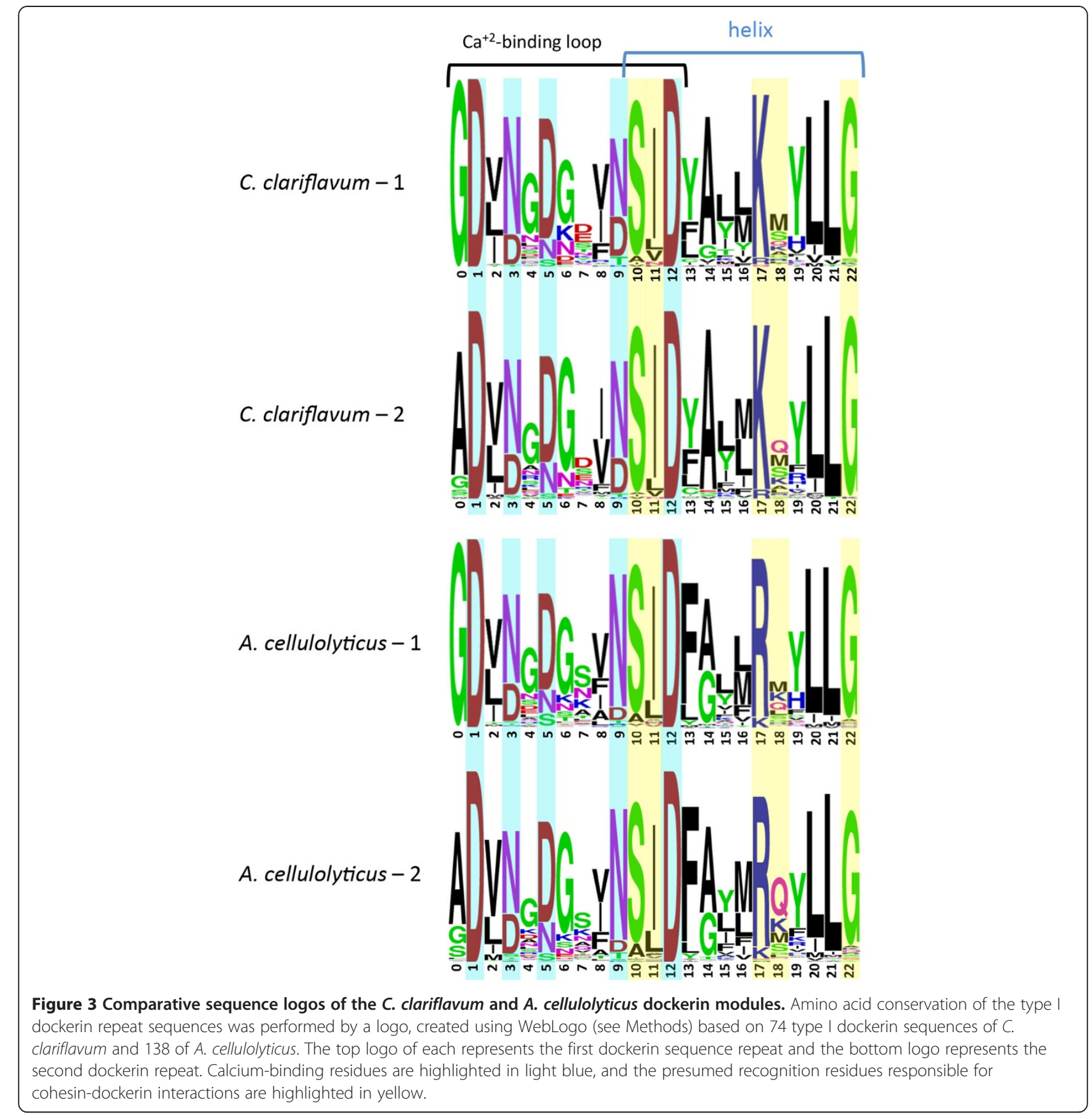

The three dockerins from the scaffoldin proteins were chosen in order to identify interactions between the different scaffoldins and to determine how the cellulosome assembles. The dockerin of the GH48 enzyme was selected as generally representative of the cellulosomal enzymes that are believed to bind type I cohesins (Figure 3). The GH48 enzyme of C. clariflavum exhibits high sequence similarity to the Cel48S enzyme from $C$. thermocellum [31] that was found to be the most abundant enzyme in the $C$. thermocellum cellulosome [14,49-52]. It seems likely that due to its abundance, the GH48 dockerin would interact with the vast repertoire of type I $C$. clariflavum cohesins. In this context, the putative recognition residues are consistent with the dominant residues shown in Figure 3.

\section{Characterization of cohesin-dockerin interactions}

All three ScaA cohesins examined in this work exhibited significant interaction with the XynDocGH48 (Figure 5), whereas the other XynDocs that were tested did not bind the ScaA cohesins significantly (that is, below the detection threshold shown in Figure 5). The type I 


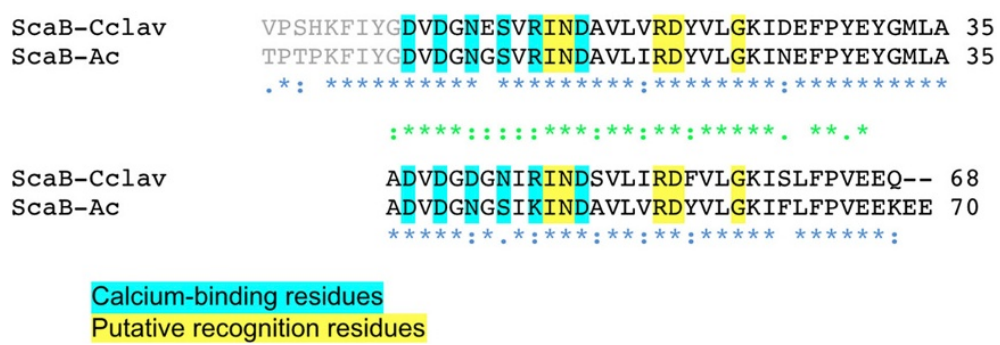

Figure 4 Sequences of $\boldsymbol{C}$. clariflavum and $\boldsymbol{A}$. cellulolyticus ScaB dockerins. Sequence alignment of the two dockerin modules was performed using the CLUSTALW2 program at the EBI website. Consensus residues are as defined accordingly; *indicates a position which has an identical residue, and colon (:) and period (.) indicate conservation between groups of strongly and weakly similar properties, respectively; blue indicates conservation between species and green indicates conservation between the two repeated segments. $\mathrm{Ca}^{+2}$-binding residues are highlighted in cyan, and putative recognition residues are highlighted in yellow. Residues are numbered relative to the highly conserved glycine (designated 0), which is positioned adjacent to the initial calcium-binding aspartate (residue 1).

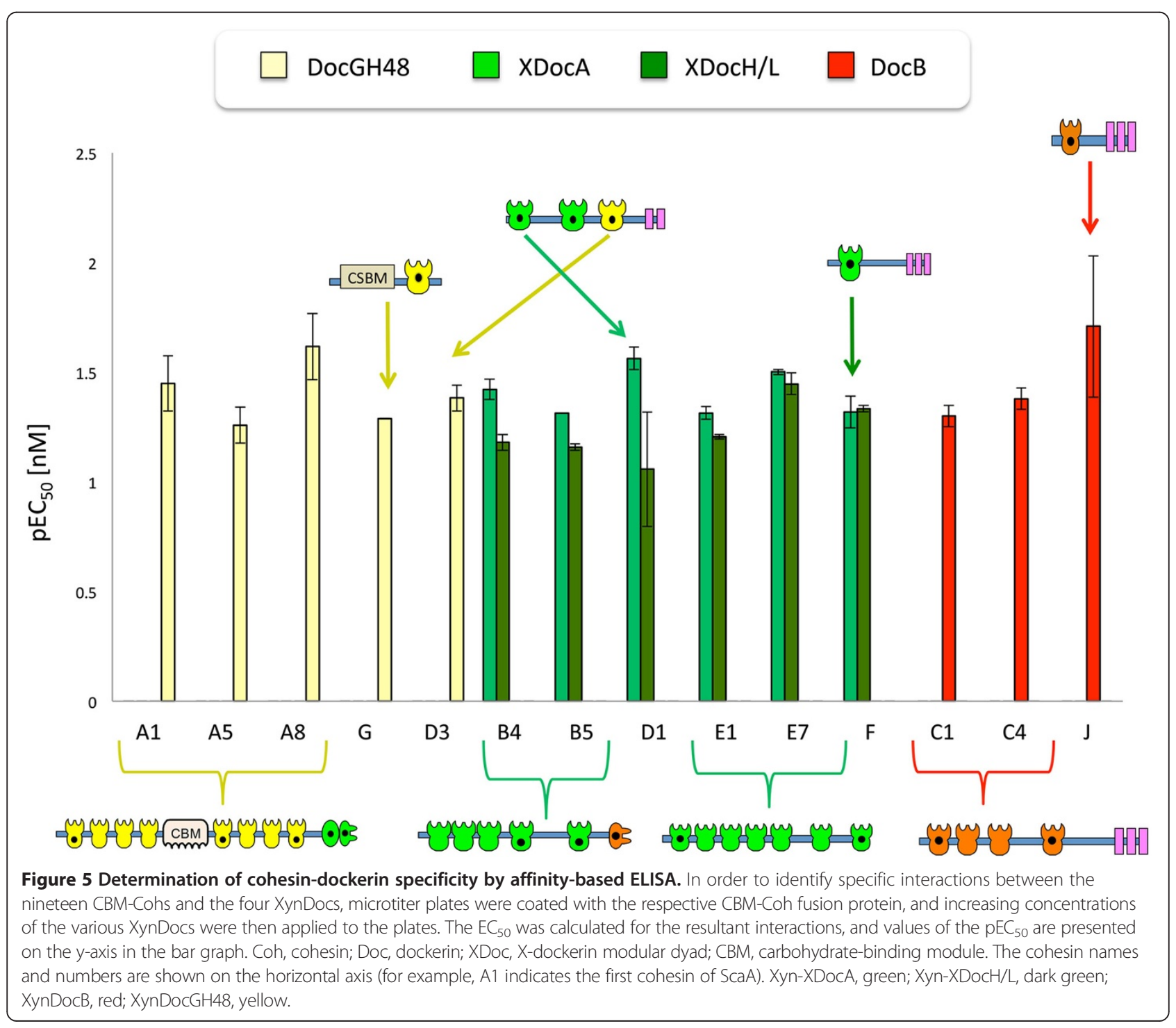


cohesin, CohG, interacted with the XynDocGH48 similarly to the ScaA CBM-Cohs.

The $\mathrm{ScaB}$ cohesins were classified bioinformatically as type II cohesins and were thus expected to interact with type II dockerins. The ELISA results verified this anticipated result, and the strongest interaction was detected between CBM-Cohs B4 and B5 and Xyn-XDocA, which also displayed a strong interaction with the $\mathrm{Xyn}-\mathrm{XDocH} /$ L. The interactions detected for the type II cohesins of ScaE (cohesins E1 and E7) and ScaF further conform to this rule. It thus appears that the type II cohesins generally interact with the type II dockerins in this organism.

Like the ScaA cohesins, the cohesins of ScaC were classified as type I cohesins. Nevertheless, CBM-Cohs $\mathrm{C} 1$ and $\mathrm{C} 4$ failed to interact with the type I XynDocGH48 but did interact with the type I XynDocB. These results correspond with the findings described by $\mathrm{Xu}$ et al. 2003 [22], for ScaB [ZP_09464032] and ScaC [ZP_09464031] from $A$. cellulolyticus, and anticipated by the status of the putative recognition residues. In this context, the residues of the ScaB dockerin (Figure 4) are very different from those of the enzyme-borne dockerins (Figure 3). As mentioned above, the recognition sequences of the ScaB dockerins from both species are identical, and it is thus not surprising that interspecies cross-reactivity was observed between representative $\mathrm{ScaC}$ cohesins and the ScaB dockerins (data not shown).

ScaJ was also classified as type I cohesin, and, like the $\mathrm{ScaC}$ cohesins, its CBM-Coh interacted exclusively with XynDocB. This specificity pattern for the ScaB dockerin reflects the phylogenetic status of the cohesins. The cohesin modules of ScaC and ScaJ from both C. clariflavum and A. cellulolyticus are located on a separate branch of the type I cohesins in the phylogenetic tree (Figure 2). These findings, together with the pattern of interactions that these modules display, suggest that $\mathrm{ScaC}$ and ScaJ belong to a subtype of cohesin modules that is distinct from the rest of the type I modules.

The unique scaffoldin $\mathrm{ScaD}$, which according to its gene sequence bears 2 type II cohesins (cohesins D1 and D2) and one type I cohesin (cohesin D3), was shown to bind two different types of dockerins. CBM-CohD1 showed the strongest interaction with Xyn-XDocA and a moderately high interaction with $\mathrm{Xyn}-\mathrm{XDocH} / \mathrm{L}$. However, CBMCohD3 exhibited type-specific binding to XynDocGH48. These results are compatible with the work of $\mathrm{Xu}$ et al. 2004 [37], which have shown the same pattern of binding for ScaD [ZP_09464030] from A. cellulolyticus. The existence of two different types of cohesins renders ScaD as both a primary scaffoldin (binds enzymes) and anchoring scaffoldin (binding other scaffoldins to the cell surface). The presence of this singular type of scaffoldin in both $C$. clariflavum and $A$. cellulolyticus appears to be a defining feature of these two cellulosome-producing species.
Five CBM-Cohs $(\mathrm{H} / \mathrm{L} 1, \mathrm{H} / \mathrm{L} 2, \mathrm{H} / \mathrm{L} 3, \mathrm{D} 2$ and $\mathrm{O})$ that were examined showed no significant binding to either of the XynDocs, using the affinity-based ELISA approach. Although CBM-Cohs H/L1, H/L2, H/L3 and O do not show strong interactions with any of the dockerins, they had a preferential but very weak binding to XynDocGH48 (below the threshold shown in the graph). Moreover, CBM-CohD2 also failed to show detectable interactions with any of the XynDocs. In order to examine whether this was related to the concentration of the reactants, we increased the coating concentration of the CBM-Cohs at $25 \mathrm{nM}$ of CBM-CohD2, which then promoted a significant interaction between CBM-CohD2 and Xyn-XDocA and $\mathrm{Xyn}-\mathrm{XDocH} / \mathrm{L}$, thus suggesting a weaker but specific cohesin-dockerin interaction in these cases. There remains the possibility that the CBM-CohD2 might be relatively unstable and subject to misfolding or denaturation, which would also account for the observed results.

\section{Discussion}

As fossil fuel reserves are exhausted, the industrialized world will require large supplies of renewable energy resources in order to maintain the current quality of life without consuming the natural energy supplies on earth. Recycling of dedicated biofuel crops and biomass waste will become an essential and primary part of the solution for future energy demands. Many cellulolytic bacteria capable of degrading polysaccharide substrates have been discovered and explored [6,53-58], and research into new cellulolytic species will enrich our knowledge of ecofriendly biomass degradation. Owing to the purported efficient cellulolytic properties of cellulosomes, the characterization of the relatively limited number of bacteria that produce them is of special interest. Progress in the field will thus pave the way for industrialized use of cellulolytic enzymes in green energy production.

The exploration of the cellulosomal genes of C. clariflavum has revealed a modular protein construction set that allows the assembly of intricate multi-enzyme architectures. This structural and enzymatic complexity is likely a key to the bacterium's reported highly efficient cellulosedegradation capabilities [29]. We investigated the putative cohesin- and dockerin-containing genes and identified 13 scaffoldins, which contain a variety of modules and domains that are distributed among the 13 polypeptide chains. C. clariflavum and A. cellulolyticus show high sequence homology [31] and display similar scaffoldin architectures and high homology among their cohesin modules (Figure 2). Similarly, the type I dockerin sequences of C. clariflavum are closely related to A. cellulolyticus dockerins and exhibit similar cohesin recognition residues. In contrast to this remarkable similarity, the glycoside hydrolases of C. clariflavum show high homology to those of C. thermocellum rather than A. cellulolyticus [31]. As can 
be seen from these findings, $C$. clariflavum appears to have acquired characteristics from both $C$. thermocellum and A. cellulolyticus. In this context, it is a thermophilic bacterium like $C$. thermocellum and has an exceptionally complex set of cellulosomal components like A. cellulolyticus $[29,31,34]$.

The detected cohesin-dockerin interactions from the affinity-ELISA studies suggest a large number of possible cellulosome architectures. Figure 6 shows the many possible complexes that can be formed. Based on the interactions among the cellulosomal components of this study, one can appreciate the complexity of such complexes by examining the interconnectivities possible for ScaC. ScaC has three SLH domains, which would together serve to anchor the complex to the cell surface, and four cohesins that have the ability to bind four ScaB dockerins. $\mathrm{ScaB}$, in turn, bears five cohesins and can thus bind the XDoc modular dyad of ScaA. ScaA can then bind multiple type I dockerins, borne by the various $C$. clariflavum enzymes. Such a complex, with fully occupied cohesins, would thus theoretically include 160 enzymatic units, thus rendering the multi-enzyme $C$. clariflavum system the largest cellulosomal complex yet discovered, superseding the deduced architectures of $C$. thermocellum (63 enzymes), A. cellulolytics (96 enzymes) and B. cellulosolvens (110 enzymes). Similarly, a cellulosome constructed of ScaJ as the anchoring scaffoldin would create a complex with 40 enzymatic units, a cellulosome built with $\mathrm{ScaD}$ as its anchoring scaffoldin would represent a complex of 17 enzymes, and a cellulosome based on ScaF would result in a complex of 8 enzymes - all having ScaA as the primary scaffoldin. The remarkable diversity of these cell-bound cellulosome assemblies in C. clariflavum mirrors that of the A. cellulolyticus assemblies, as they have a homologous set of anchoring scaffoldins. This diversity appears to reflect the elaborate surface morphology observed previously for A. cellulolyticus.

The ScaE-based cellulosomal complex appears to be the only potential cell-free cellulosome system in this bacterium that would catalyze plant cell wall polysaccharides independent of the location of the bacterial cell and may thus enhance the decomposition of recalcitrant substrates into simpler oligosaccharide units. ScaE is composed of seven type II cohesins and has no dockerin module, SLH domain, CBM module, or other detectable sequence that would anchor it to the cell surface or to the polysaccharide substrate. However, its seven type II cohesins are capable of binding the XDoc modules of ScaA. A cellulosome constructed of ScaE and 7 ScaAs would contain 56 enzymes and would supply 7 CBM3

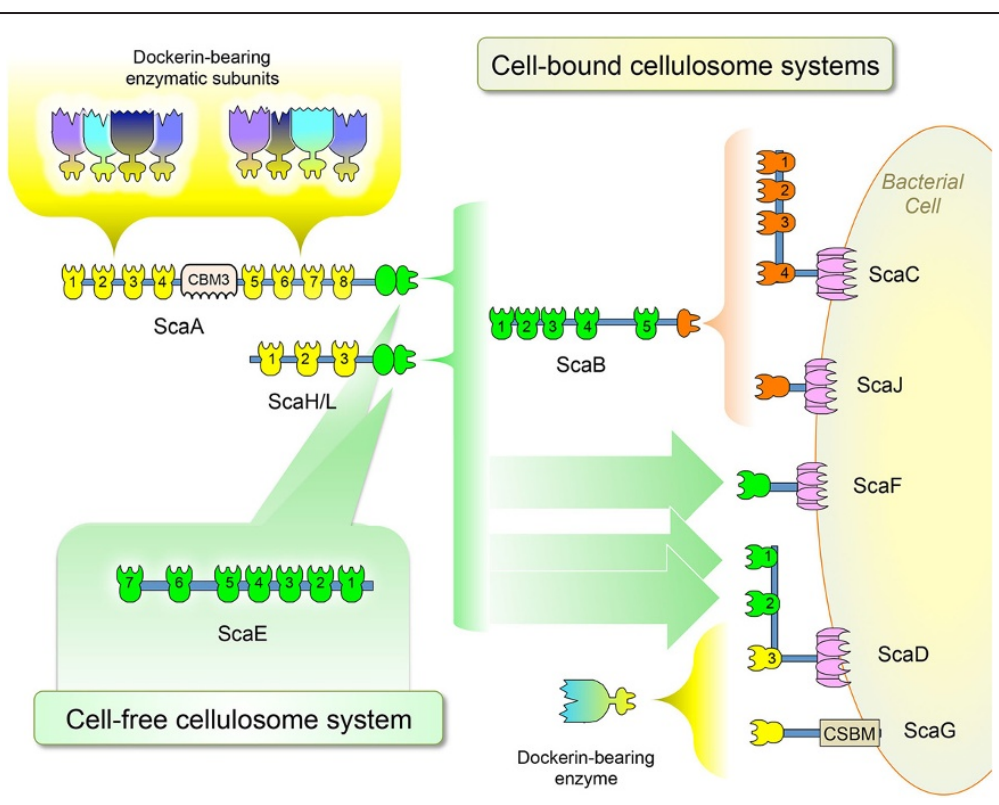

Figure 6 Proposed architectures for cell-bound and cell-free cellulosome assembly in C. clariflavum. The scheme shows the possible interactions among scaffoldin and enzymatic modules, as derived from examination of interactions by affinity ELISA. Specification of scaffoldins is detailed in Figure 1. Four potential cell-anchored cellulosomal complexes are represented. Two of the complexes employ the adaptor protein ScaB to join the cell-anchored scaffoldins (ScaC and ScaJ, containing an SLH domain, and four and one type I cohesins, respectively) to the primary enzyme-integrating scaffoldins (ScaA and ScaH/L) via the type II cohesins of ScaB and XDocs of the former. The type II cohesins of ScaD (cohesins 1 and 2) and ScaF are also cell-anchored scaffoldins that bind directly ScaA or ScaH/L. The type I cohesins of ScaG and ScaD (cohesin 3) interact with type I dockerins of dockerin-bearing enzymes. ScaG is suspected to be a cell-anchored scaffoldin, based on previous studies of the copper amine-oxidase domain in the OlpC protein from C. thermocellum. ScaE has seven type II cohesins which are able to bind seven XDoc modules, thereby creating a large, cell-free cellulosomal complex. CBSM, cell surface-binding module. 
modules, which can direct the complex to substrate. This type of scaffoldin is also produced in both A. cellulolyticus and C. thermocellum, that is, ScaE [34] and Cthe_0736 [16], respectively - both of which also contain seven type II cohesins. The presence of this type of scaffoldin in these three bacteria emphasizes its apparent importance in complex cellulosome systems. In contrast, the simple cellulosome systems of mesophilic clostridia, such as $C$. cellulolyticum, C. cellulovorans and C. papyrosolvens, all contain a single primary scaffoldin alone and lack scaffoldins that bear type II cohesins [12,59].

It is important to note that all the type II cohesins examined in the current study have shown interaction with the XDoc of both ScaA and ScaH/L. Combined interaction with the two scaffoldins allows for a large number of possible cellulosome assemblies. Nevertheless, no significant interaction was detected between the ScaH/L cohesins and the tested dockerins, although these cohesins may interact with other dockerin-containing enzymes which were not tested in this work. In order to expand our knowledge of the specificity of dockerin-containing enzymes incorporated into the cellulosome, more dockerin modules from C. clariflavum genome will be investigated in the future.

\section{Conclusions}

In this work we revealed a novel, complicated, intriguing cellulosomal system that has the potential to help us understand the cellulosomal conversion of recalcitrant polysaccharide substrates to simple sugars and their subsequent conversion to biofuels. The multiplicity of cellulosomal components in C. clariflavum and their possible interactions and interconnectivities observed gives rise to the formation of diverse complexes that enable efficient cellulose degradation. Furthermore, the stability of C. clariflavum proteins is expected to be higher in comparison to mesophilic bacteria such as A. cellulolyticus, as C. clariflavum is a thermophilic bacterium [29-31]. Until the discovery of a cellulosome system in C. clariflavum, the only thermophile known to produce a cellulosome system has been $C$. thermocellum. Our findings suggest that $C$. clariflavum can be further developed into a good source for new potent cellulose-degrading enzymes and novel cellulosomal architectures, thus providing a thermophilic cellulosomeproducing alternative to the prototypical $C$. thermocellum system.

\section{Materials and methods Genomes source}

Genome sequences of C. clariflavum DSM 19732 [CP003065], A. cellulolyticus CD2 [NZ AEDB02000000], and C. thermocellum ATCC 27405 [CP000568] were obtained from the GenBank of NCBI [60].

\section{Sequence analysis and database searches}

BLASTP algorithm [61] searches were performed for predicted proteins of C. clariflavum, using deduced amino acid sequences of the known cohesin and dockerin modules as queries. Hits above an E-value of $10^{-4}$ were examined individually, by searching for characteristic sequence features. For example, for dockerin modules, we searched for two $\mathrm{Ca}^{+2}$-binding repeats, putative helices and linker regions.

Multiple sequence alignments (MSAs) were created using the CLUSTALW servers, at the EBI [35] and at the ExPASy Website [62]. When needed, MSAs generated by the EBI CLUSTALW2 server were used to reconstruct phylogenetic trees in the MEGA 5.10 software [36] using the neighbor-joining method with 500 bootstrap replicates. Amino acid sequence logos were performed using the WebLogo application, version 2.8.2 [63].

\section{Annotation of dockerin-containing enzymes}

In order to identify and analyze enzymatic modules of the dockerin-containing proteins of C. clariflavum DSM 19732, the proteins were annotated using the Carbohydrate Active Enzymes database (CAZY) [64,65]. The analysis was based on sequence conservation between catalytic modules, and the different catalytic modules were sorted into different family types, such as GHs, glycosyltransferases, PLs, CEs and CBMs.

\section{Source of C. clariflavum genomic DNA}

C. clariflavum DSM 19732 was supplied by the Leibniz Institute DSMZ-German Collection of Microorganisms and Cell Cultures. The bacterium was grown on GS-2 medium. Genomic DNA was extracted by Dr Harish Kumar Reddy (Tel Aviv University), as described earlier [66].

\section{Cloning and design of CBM3a-Cohesin plasmid cassettes}

The pET28a plasmid was used to create fusion proteins CBM3a-Cohesin (CBM-Coh). The PCR product of the CBM3a module from $C$. thermocellum scaffoldin CipA [26] was inserted into the pET28a plasmid by using NcoI and BamHI sites as previously described by Barak et al. 2005 [47]. Genes encoding cohesin modules were cloned using specific primers (Additional file 3: Table S1) by PCR from C. clariflavum genomic DNA using Reddymix $x 2$ (Advanced Biotechnologies Ltd., Epsom, Surrey, United Kingdom). The amplified DNA fragments were purified by The HiYield gel-PCR fragment extraction kit (Real Biotech Corporation, RBC, Banqiao City, Taiwan). Cohesin inserts were restricted by BamHI ( 5 ' terminus) and XhoI (3' terminus) FastDigest enzymes (Thermo scientific, Fermentas UAB, Vilnius, Lithuania) and ligated into the pET28a-CBM3a cassette $[40,47]$. The plasmids were transformed into an E. coli XL-1 Blue strain and purified 
via QIAprep spin miniprep kit (QIAGEN GmbH, D-40724 Hilden, Germany).

\section{Xylanase-dockerin (Xyn-Doc) cassettes}

A PCR product of G. stearothermophilus T6 xylanase with a His-tag and $\mathrm{BspHI}$ (5' terminus) and $\mathrm{KpnI}$ ( 3 ' terminus) restriction sites was obtained $[40,47,48,67]$ and inserted into the pET9d vector. The dockerin modules were produced using specific primers (supplementary material) by PCR with the KpnI site at the $5^{\prime}$ terminus and the BamHI at the 3 ' terminus. The dockerin-encoding genes were inserted into the plasmid using $\mathrm{KpnI}$ and BamHI enzymes.

\section{Protein expression}

The pET28a cassette containing the CBM-Coh fusion proteins and the pET9d cassette containing the XynDoc fusion proteins were transformed into E. coli BL21 (DE3) strains and plated onto LB-kanamycin plates. For each plate, 4 to $5 \mathrm{~mL}$ of Luria-Bertani broth (LB) were added in order to resuspend the cells. The cells were added to $1 \mathrm{~L}$ of LB with $50 \mu \mathrm{g} / \mathrm{mL}$ kanamycin (Sigma-Aldrich, Rehovot, Israel) and $2 \mathrm{mM} \mathrm{CaCl}_{2}$ and were grown for $2.5 \mathrm{~h}$ at $37^{\circ} \mathrm{C}$ to $\mathrm{A}_{600} \approx 0.8$ to 1.0. Induction for protein expression was made by adding Isopropyl-1-thio- $\beta$-D-galactoside (IPTG) (Fermentas UAB, Vilnius, Lithuania) in a final concentration of $0.2 \mathrm{mM}$, and the growth was continued in $16^{\circ} \mathrm{C}$ for $16 \mathrm{~h}$. Cells were harvested by centrifugation at $5,000 \mathrm{rpm}$ for 15 minutes.

\section{CBM-Coh purification}

After centrifugation, cells were resuspended with $30 \mathrm{~mL}$ TBS (Tris-buffered saline, $137 \mathrm{mM} \mathrm{NaCl}, 2.7 \mathrm{mM} \mathrm{KCL}$, $25 \mathrm{mM}$ Tris- $\mathrm{HCl}, \mathrm{pH}=7.4)$, and protease-inhibitor cocktail was added (1 mM PMSF, $0.4 \mathrm{mM}$ benzamidine and $0.06 \mathrm{mM}$ benzamide). The cells were sonicated and the supernatant was centrifuged for 30 minutes at 15,000 $\mathrm{rpm}$ at $4^{\circ} \mathrm{C}$. The supernatant was then added to $2 \mathrm{~g}$ of macroporous bead cellulose preswollen gel (IONTOSORB, usti nad Labem, Czech Republic) and incubated for $1 \mathrm{~h}$, with rotation at $4^{\circ} \mathrm{C}$. The mixture was then loaded onto a gravity column and washed with $100 \mathrm{~mL}$ of TBS that contained $1 \mathrm{M} \mathrm{NaCl}$, and then washed with $100 \mathrm{~mL}$ TBS. Three $10-\mathrm{mL}$ elutions of $1 \%$ triethanolamine (TEA) were then collected. The three fractions were subjected to SDS-PAGE in order to assess protein purity, and then dialyzed with TBS containing $5 \mathrm{mM}$ $\mathrm{CaCl}_{2}$.

\section{Xyn-Doc purification}

After centrifugation, cells were resuspended with $30 \mathrm{~mL}$ TBS supplemented with $5 \mathrm{mM}$ imidazole and proteaseinhibitor cocktail. Cells were disrupted by sonication and centrifuged for 30 minutes at $15,000 \mathrm{rpm}$ at $4^{\circ} \mathrm{C}$.
The purification was performed in a batch purification system as described previously by Vazana et al. 2010. Fractions of $2 \mathrm{~mL}$ were collected, and protein purity was assessed by SDS-PAGE. The fractions that contained the protein were pooled and dialyzed with TBS and $5 \mathrm{mM}$ $\mathrm{CaCl}_{2}$.

\section{Protein concentration and storage}

Proteins concentrations were evaluated by absorbance at $280 \mathrm{~nm}$, based on the extinction coefficients derived from the known composition of amino acids of each protein. Extinction coefficients were calculated using the ExPASy ProtParam tool [68]. The proteins were concentrated by Amicon ultra concentrators (Millipore, Carrigtwohill, Co. Cork, Ireland), and stored at $-20^{\circ} \mathrm{C}$ in $50 \%$ (vol/vol) glycerol.

Affinity-based ELISA was performed by the protocol reported earlier by Barak et al. 2005 [47]. The 96-well ELISA plates (Nunc, A/S, Roskilde, Denmark) were coated with the fusion proteins CBM-Cohs at a concentration of $3 \mathrm{nM}$, and variable concentrations of Xyn-Docs (ranging between $2 \mathrm{pM}$ and $20 \mathrm{nM}$ ) were used to detect specific cohesin-dockerin interactions. The interactions with the four XynDocs proteins were examined immunochemically by using anti-xylanase primary antibody and horseradish peroxidase (HRP)-labeled secondary antibody. For comparative purposes, $\mathrm{pEC}_{50}$ was calculated for each binding curve as described earlier $[47,69]$ and the results were presented in bar graph form.

\section{Additional files}

Additional file 1: Figure S1. Multiple sequence alignment of 106 cohesin sequences originated from the genomes of C. clariflavum (Cc), $A$. cellulolyticus (AC) and C. thermocellum (Ct). Alignment length: 148; identity $(*): 1$ residue $=0.67 \%$; strongly similar $(:): 3$ residues $=2.03 \%$; weakly similar (.): 3 residues $=2.03 \%$; different: 141 residues $=95.27 \%$. All the accession numbers for $C$. clariflavum cohesin-containing proteins can be found in Figure 1, and the accession numbers of A. cellulolyticus and C. thermocellum cohesin-containing proteins can be found in Dassa et al. 2012 [34].

Additional file 2: Figure S2. Multiple sequence alignment of the $C$. clariflavum 74 dockerin modules. Cyan highlight indicates putative calcium-binding residues. Yellow highlight indicates putative recognition residues. Gray highlight marks the last C-terminal residue of a corresponding protein. $x$ indicates a computational fusion of Clocl_2272 [YP_005046783] and Clocl_2271 [YP_005046782] to reconstruct a complete dockerin motif (a stop codon TAA of Clocl_2272 was replaced with NNN). BIL, bacterial intein-like domain; CARDB, cell adhesion-related domain found in bacteria; CBM, carbohydrate binding module (followed by family number); $C E$, carbohydrate esterase (followed by family number); $\mathrm{COH}$, cohesin; DOC, dockerin; EXPN, expansin; FN3, fibronectin type III domain; GH, glycoside hydrolase (followed by family number); LNK, linker; $\mathrm{PL}$, polysaccharide lyase (followed by family number); Serpin, serine protease inhibitor; SIGN, signal peptide; UNK, unknown region; $X, X$ domain. Alignment length: 84. Identity $\left(^{*}\right): 5$ identical residues $=5.62 \%$. Strongly similar (:): 1 residue $=1.12 \%$. Weakly similar (.): 3 residues $=3.37 \%$. Different: 80 residues $=89.89 \%$.

Additional file 3: Table S1. List of primers for the C. clariflavum cohesin and dockerin modules that were cloned in this study. Nucleotides shown in bold indicate restriction sites added to the primers. 


\section{Abbreviations}

CBM: carbohydrate-binding module; CE: carbohydrate esterase; Coh: cohesin; CSBM: cell surface-binding module; Doc: dockerin; ELISA: enzyme-linked immunosorbent assay; GH: glycoside hydrolase; MSA: multiple sequence alignment; ORF: open reading frame; PL: polysaccharide lyase; SLH: S-layer homology; XDoc: X module coupled with a type II dockerin; Xyn: xylanase.

\section{Competing interests}

The authors declare that they have no competing interests.

\section{Authors' contribution}

$\angle A$ and $E A B$ designed the research. $L A$ and MS performed the experiments. $L A, R L$ and $E A B$ analyzed the results. $B D$ and $I B$ analyzed the genome data. $L A, B D$ and $E A B$ wrote the manuscript. All authors read and approved the manuscript.

\section{Acknowledgements}

This research was supported by the establishment of an Israeli Center of Research Excellence (I-CORE Center number 152/11, EAB and YS) managed by the Israel Science Foundation, from the United States-Israel Binational Science Foundation (BSF), Jerusalem, Israel, by the Weizmann Institute of Science Alternative Energy Research Initiative (AERI) and the Helmsley Foundation, and a grant to $E A B$ and $R L$ from the Israel Ministry of Science (IMOS). The authors appreciate the support of the European Union, Area NMP.2013.1.1-2: Self-assembly of naturally occurring nanosystems, project number 604530. Additional support was obtained from a grant (number 1349) to EAB also from the Israel Science Foundation (ISF) and a grant (number 24/11) issued to RL by The Sidney E. Frank Foundation also through the ISF. This research was also supported by a grant from the F. Warren Hellman Grant for Alternative Energy Research in Israel in support of alternative energy research in Israel to EAB administered by the Israel Strategic Alternative Energy Foundation (I-SAEF). EAB is the incumbent of The Maynard I. and Elaine Wishner Chair of Bio-organic Chemistry.

\section{Author details}

'Department of Biological Chemistry, The Weizmann Institute of Science, Rehovot, Israel. ${ }^{2}$ Department of Molecular Microbiology and Biotechnology, Tel Aviv University, Ramat Aviv, Israel.

Received: 12 March 2014 Accepted: 12 June 2014

Published: 1 July 2014

\section{References}

1. Bayer EA, Lamed R: The cellulose paradox: pollutant par excellence and/or a reclaimable natural resource? Biodegradation 1992, 3:171-188.

2. Bayer EA, Lamed R, Himmel ME: The potential of cellulases and cellulosomes for cellulosic waste management. Curr Opinion Biotechnol 2007, 18:237-245.

3. Gilbert $\mathrm{HJ}$ : The biochemistry and structural biology of plant cell wall deconstruction. Plant Physiol 2010, 153:444-455.

4. Atalla RH: Celluloses. In Comprehensive natural products chemistry, Volume Volume 3. Edited by Pinto BM. Cambridge: Elsevier; 1999:529-598.

5. O'Sullivan AC: Cellulose: the structure slowly unravels. Cellulose 1997, 4:173-207

6. Bayer EA, Shoham Y, Lamed R: Lignocellulose-decomposing bacteria and their enzyme systems. In The Prokaryotes. 4th edition. Edited by Rosenberg E. Berlin: Springer; 2013:216-266.

7. Demain AL, Newcomb M, Wu JH: Cellulase, clostridia, and ethanol. Microbiol Mol Biol Rev 2005, 69:124-154.

8. Ragauskas AJ, Williams CK, Davison BH, Britovsek G, Cairney J, Eckert CA, Frederick WJ Jr, Hallett JP, Leak DJ, Liotta CL, Mielenz JR, Murphy R, Templer $R$, Tschaplinski $T$ : The path forward for biofuels and biomaterials. Science 2006, 311:484-489.

9. Himmel ME, Ding S-Y, Johnson DK, Adney WS, Nimlos MR, Brady JW, Foust TD: Biomass recalcitrance: Engineering plants and enzymes for biofuels production. Science 2007, 315:804-807. Erratum: 316, 982.

10. Bayer EA, Morag E, Lamed R: The cellulosome - A treasure-trove for biotechnology. Trends Biotechnol 1994, 12:379-386.

11. Bayer EA, Chanzy H, Lamed R, Shoham Y: Cellulose, cellulases and cellulosomes. Curr Opin Struct Biol 1998, 8:548-557.
12. Bayer EA, Belaich J-P, Shoham Y, Lamed R: The cellulosomes: Multi-enzyme machines for degradation of plant cell wall polysaccharides. Annu Rev Microbiol 2004, 58:521-554

13. Bayer EA, Kenig R, Lamed R: Adherence of Clostridium thermocellum to cellulose. J Bacteriol 1983, 156:818-827.

14. Lamed R, Setter E, Bayer EA: Characterization of a cellulose-binding, cellulase-containing complex in Clostridium thermocellum. J Bacteriol 1983, 156:828-836.

15. Lamed R, Setter E, Kenig R, Bayer EA: The cellulosome - a discrete cell surface organelle of Clostridium thermocellum which exhibits separate antigenic, cellulose-binding and various cellulolytic activities. Biotechnol Bioeng Symp 1983, 13:163-181.

16. Fontes CM, Gilbert HJ: Cellulosomes: Highly efficient nanomachines designed to deconstruct plant cell wall complex carbohydrates. Annu Rev Biochem 2010, 79:655-681.

17. Gerngross UT, Romaniec MPM, Kobayashi T, Huskisson NS, Demain AL: Sequencing of a Clostridium thermocellum gene (cipA) encoding the cellulosomal $\mathrm{S}_{\mathrm{L}}$-protein reveals an unusual degree of internal homology. Mol Microbiol 1993, 8:325-334.

18. Kakiuchi M, Isui A, Suzuki K, Fujino T, Fujino E, Kimura T, Karita S, Sakka K, Ohmiya K: Cloning and DNA sequencing of the genes encoding Clostridium josui scaffolding protein CipA and cellulase CelD and identification of their gene products as major components of the cellulosome. J Bacteriol 1998, 180:4303-4308.

19. Pagès S, Belaich A, Fierobe H-P, Tardif C, Gaudin C, Belaich J-P: Sequence analysis of scaffolding protein CipC and ORFXp, a new cohesin-containing protein in Clostridium cellulolyticum: comparison of various cohesin domains and subcellular localization of ORFXp. J Bacteriol 1999, 181:1801-1810.

20. Shoseyov O, Takagi M, Goldstein MA, Doi RH: Primary sequence analysis of Clostridium cellulovorans cellulose binding protein A. Proc Natl Acad Sci USA 1992, 89:3483-3487.

21. Ding S-Y, Bayer EA, Steiner D, Shoham Y, Lamed R: A novel cellulosomal scaffoldin from Acetivibrio cellulolyticus that contains a family-9 glycosyl hydrolase. J Bacterio/ 1999, 181:6720-6729.

22. Xu Q, Gao W, Ding S-Y, Kenig R, Shoham Y, Bayer EA, Lamed R: The cellulosome system of Acetivibrio cellulolyticus includes a novel type of adaptor protein and a cell-surface anchoring protein. J Bacteriol 2003, 185:4548-4557.

23. Lemaire $M$, Ohayon H, Gounon P, Fujino T, Béguin P: OlpB, a new outer layer protein of Clostridium thermocellum, and binding of its S-layer-like domains to components of the cell envelope. J Bacterio/ 1995, 177:2451-2459.

24. Lupas A, Engelhardt H, Peters J, Santarius U, Volker S, Baumeister W: Domain structure of the Acetogenium kivui surface layer revealed by electron crystallography and sequence analysis. J Bacteriol 1994 176:1224-1233.

25. Poole DM, Morag E, Lamed R, Bayer EA, Hazlewood GP, Gilbert HJ: Identification of the cellulose binding domain of the cellulosome subunit S1 from Clostridium thermocellum. FEMS Microbiol Lett 1992, 99:181-186.

26. Morag E, Lapidot A, Govorko D, Lamed R, Wilchek M, Bayer EA, Shoham Y: Expression, purification and characterization of the cellulose-binding domain of the scaffoldin subunit from the cellulosome of Clostridium thermocellum. Appl Environ Microbiol 1995, 61:1980-1986.

27. Shoseyov O, Shani Z, Levy I: Carbohydrate binding modules: biochemical properties and novel applications. Microbiol Mol Biol Rev 2006, 70:283-295.

28. Boraston AB, Bolam DN, Gilbert HJ, Davies GJ: Carbohydrate-binding modules: fine-tuning polysaccharide recognition. Biochem J 2004, 382(Pt 3):769-781.

29. Shiratori H, Ikeno H, Ayame S, Kataoka N, Miya A, Hosono K, Beppu T, Ueda $\mathrm{K}$ : Isolation and characterization of a new Clostridium sp. that performs effective cellulosic waste digestion in a thermophilic methanogenic bioreactor. Appl Environ Microbiol 2006, 72:3702-3709.

30. Shiratori H, Sasaya K, Ohiwa H, Ikeno H, Ayame S, Kataoka N, Miya A, Beppu T, Ueda K: Clostridium clariflavum sp. nov. and Clostridium caenicola sp. nov., moderately thermophilic, cellulose-/cellobiose-digesting bacteria isolated from methanogenic sludge. Int J Syst Evol Microbiol 2009, 59(Pt 7):1764-1770.

31. Izquierdo JA, Goodwin L, Davenport KW, Teshima H, Bruce D, Detter C, Tapia R, Han S, Land M, Hauser L, Jeffries CD, Han J, Pitluck S, Nolan M, Chen A, Huntemann M, Mavromatis K, Mikhailova N, Liolios K, Woyke T, Lynd LR: Complete genome sequence of Clostridium clariflavum DSM 19732. Stand Genomic Sci 2012, 6:104-115. 
32. Khan AW: Cellulolytic enzyme system of Acetivibrio cellulolyticus, a newly isolated anaerobe. J Gen Microbiol 1980, 121:499-502

33. Patel GB, Khan AW, Agnew BJ, Colvin JR: Isolation and characterization of an anaerobic cellulolytic microorganism, Acetivibrio cellulolyticus, gen. nov., sp. nov. Int J Syst Bacteriol 1980, 30:179-185.

34. Dassa B, Borovok I, Lamed R, Henrissat B, Coutinho P, Hemme CL, Huang Y, Zhou Z, Bayer EA: Genome-wide analysis of Acetivibrio cellulolyticus provides a blueprint of an elaborate cellulosome system. BMC Genomics 2012, 13:210.

35. ClustalW2 - multiple sequence alignment program for DNA or proteins. http://www.ebi.ac.uk/Tools/msa/clustalw2/

36. Tamura K, Stecher G, Peterson D, Filipski A, Kumar S: MEGA6: Molecular Evolutionary Genetics Analysis Version 6.0. Mol Biol Evol 2013, 30:2725-2729

37. Xu Q, Barak Y, Kenig R, Shoham Y, Bayer EA, Lamed R: A novel Acetivibrio cellulolyticus anchoring scaffoldin that bears divergent cohesins. J Bacteriol 2004, 186:5782-5789.

38. Adams JJ, Webb BA, Spencer HL, Smith SP: Structural characterization of type II dockerin module from the cellulosome of Clostridium thermocellum: Calcium-induced effects on conformation and target recognition. Biochemistry 2005, 44:2173-2182.

39. Leibovitz E, Béguin P: A new type of cohesin domain that specifically binds the dockerin domain of the Clostridium thermocellum cellulosome-integrating protein CipA. J Bacterio/ 1996, 178:3077-3084.

40. Haimovitz R, Barak Y, Morag E, Voronov-Goldman M, Lamed R, Bayer EA Cohesin-dockerin microarray: Diverse specificities between two complementary families of interacting protein modules. Proteomics 2008, 8:968-979.

41. Gefen G, Anbar M, Morag E, Lamed R, Bayer EA: Enhanced degradation of cellulose by targeted incorporation of a cohesin-fused $\beta$-glucosidase into the Clostridium thermocellum cellulosome. Proc Natl Acad Sci USA 2012, 109:10298-10303.

42. Pinheiro BA, Gilbert HJ, Sakka K, Fernandes VO, Prates JA, Alves VD, Bolam $D N$, Ferreira $L M$, Fontes $C M$ : Functional insights into the role of novel type I cohesin and dockerin domains from Clostridium thermocellum. Biochem J 2009, 424:375-384.

43. Simpson PJ, Hefang X, Bolam DN, Gilbert HJ, Williamson MP: The structural basis for the ligand specificity of Family 2 carbohydrate binding modules. J Biol Chem 2000, 52:41137-41142.

44. Pagès $S$, Belaich A, Belaich J-P, Morag E, Lamed R, Shoham Y, Bayer EA Species-specificity of the cohesin-dockerin interaction between Clostridium thermocellum and Clostridium cellulolyticum: Prediction of specificity determinants of the dockerin domain. Proteins 1997, 29:517-527.

45. Mechaly A, Yaron S, Lamed R, Fierobe H-P, Belaich A, Belaich J-P, Shoham Y, Bayer EA: Cohesin-dockerin recognition in cellulosome assembly: Experiment versus hypothesis. Proteins 2000, 39:170-177.

46. Mechaly A, Fierobe H-P, Belaich A, Belaich J-P, Lamed R, Shoham Y, Bayer EA: Cohesin-dockerin interaction in cellulosome assembly: A single hydroxyl group of a dockerin domain distinguishes between non-recognition and high-affinity recognition. J Biol Chem 2001, 276:9883-9888. Erratum 19678.

47. Barak Y, Handelsman T, Nakar D, Mechaly A, Lamed R, Shoham Y, Bayer EA: Matching fusion-protein systems for affinity analysis of two interacting families of proteins: The cohesin-dockerin interaction. J Mol Recogit 2005, 18:491-501.

48. Lapidot A, Mechaly A, Shoham Y: Overexpression and single-step purification of a thermostable xylanase from Bacillus stearothermophilus T-6. J Biotechnol 1996, 51:259-264.

49. Wu JHD, Orme-Johnson WH, Demain AL: Two components of an extracellular protein aggregate of Clostridium thermocellum together degrade crystaline cellulose. Biochemistry 1988, 27:1703-1709.

50. Gold ND, Martin VJ: Global view of the Clostridium thermocellum cellulosome revealed by quantitative proteomic analysis. J Bacterio/ 2007, 189:6787-6795.

51. Raman B, Pan C, Hurst GB, Rodriguez M, McKeown CK, Lankford PK, Samatova NF, Mielenz JR: Impact of pretreated switchgrass and biomass carbohydrates on Clostridium thermocellum ATCC 27405 cellulosome composition: a quantitative proteomic analysis. PLOS ONE 2009, 4:e5271.

52. Dror TW, Morag E, Rolider A, Bayer EA, Lamed R, Shoham Y: Regulation of the cellulosomal celS (ce/48A) gene of Clostridium thermocellum is growth-rate dependent. J Bacterio/ 2003, 185:3042-3048.
53. Gilbert HJ (Ed): Cellulases. San Diego: Elsevier; 2012.

54. Morrison M, Daugherty SC, Nelson WC, Davidsen T, Nelson KE: The FibRumBa database: $A$ resource for biologists with interests in gastrointestinal microbial ecology, plant biomass degradation, and anaerobic microbiology. Microb Ecol 2010, 59:212-213.

55. Brown SD, Lamed R, Morag E, Borovok I, Shoham Y, Klingeman DM Johnson CM, Yang Z, Land ML, Uttukar SM, Keller M, Bayer EA: Draft genome sequences for Clostridium thermocellum wild-type strain YS and derived cellulose adhesion-defective mutant strain AD2. J Bacteriol 2012, 194:3290-3291.

56. Zepeda V, Dassa B, Borovok I, Lamed R, Bayer EA, Cate JH: Draft genome sequence of the cellulolytic bacterium Clostridium papyrosolvens $\mathrm{C} 7$ (ATCC 700395). Genome Announcements 2013, 1:e00698-13.

57. Hemme CL, Mouttaki H, Lee YJ, Zhang G, Goodwin L, Lucas S, Copeland A, Lapidus A, del Rio Glavina T, Tice H, Saunders E, Brettin T, Detter JC, Han CS, Pitluck S, Land ML, Hauser L, Kyrpides N, Mikhailova N, He Z, Wu L, Van Nostrand JD, Henrissat B, He Q, Lawson PA, Tanner RS, Lynd LR, Wiegel J, Fields MW, Arkin AP, et al: Sequencing of multiple clostridial genomes related to biomass conversion and biofuel production. J Bacterio/ 2010, 192:6494-6496.

58. Blumer-Schuette SE, Ozdemir I, Mistry D, Lucas S, Lapidus A, Cheng JF, Goodwin LA, Pitluck S, Land ML, Hauser LJ, Woyke T, Mikhailova N, Pati A, Kyrpides NC, Ivanova N, Detter JC, Walston-Davenport K, Han S, Adams MW, Kelly RM: Complete genome sequences for the anaerobic, extremely thermophilic plant biomass-degrading bacteria Caldicellulosiruptor hydrothermalis, Caldicellulosiruptor kristjanssonii, Caldicellulosiruptor kronotskyensis, Caldicellulosiruptor owensensis, and Caldicellulosiruptor lactoaceticus. J Bacteriol 2011, 193:1483-1484.

59. Doi RH, Kosugi A: Cellulosomes: plant-cell-wall-degrading enzyme complexes. Nat Rev Microbiol 2004, 2:541-551.

60. National Center for Biotechnology Information (NCBI) Website. http://www. ncbi.nlm.nih.gov/.

61. Altschul SF, Madden TL, Schäffer AA, Zhang J, Zhang Z, Miller W, Lipman DJ: Gapped BLAST and PSI-BLAST: a new generation of protein database search programs. Nucl Acids Res 1997, 25:3389-3402.

62. The EXPASy Bioinformatics Resource Portal. http://www.ch.embnet.org/ software/ClustalW.html.

63. Crooks GE, Hon G, Chandonia JM, Brenner SE: WebLogo: A sequence logo generator. Genome Res 2004, 14:1188-1190.

64. Cantarel BL, Coutinho PM, Rancurel C, Bernard T, Lombard V, Henrissat B: The Carbohydrate-Active Enzymes database (CAZy): an expert resource for glycogenomics. Nucl Acids Res 2009, 37:D233-D238.

65. Carbohydrate-Active enZYmes Database. http://www.cazy.org/.

66. Marmur J: A procedure for the isolation of deoxyribonucleic acid from micro-organisms. J Mol Biol 1961, 3:208-218.

67. Handelsman T, Barak Y, Nakar D, Mechaly A, Lamed R, Shoham Y, Bayer EA Cohesin-dockerin interaction in cellulosome assembly: A single Asp-to-Asn mutation disrupts high-affinity cohesin-dockerin binding. FEBS Lett 2004, 572:195-200.

68. ProtParam tool - computation of various physical and chemical parameters for a given protein. http://web.expasy.org/protparam/.

69. Motulsky HJ, Christopoulos A: Fitting models to biological data using linear and nonlinear regression. A practical guide to curve fitting. San Diego, CA: GraphPad Software Inc.; 2003.

doi:10.1186/1754-6834-7-100

Cite this article as: Artzi et al:: Cellulosomics of the cellulolytic

thermophile Clostridium clariflavum. Biotechnology for Biofuels 2014 7:100. 